\title{
WAVE PROPAGATION IN A 2-D OPTICAL WAVEGUIDE*
}

\author{
ROLANDO MAGNANINI ${ }^{\dagger}$ AND FADIL SANTOSA ${ }^{\ddagger}$
}

In memory of Ralph E. Kleinman (1929-1998)

\begin{abstract}
In this work, we consider a wave propagation problem in a 2-D waveguide. The problem arises in the study of light in optical fibers. We construct a transform theory as a framework for studying this problem. An explicit representation for the solution to problems involving light sources is derived. We derive a decay rate for the non-guided part of the solution in the direction of the core. The approach is also amenable to computations, and we demonstrate this in numerical examples for the case of slab waveguides.
\end{abstract}

Key words. Wave propagation, optical waveguides, Green function.

AMS subject classifications. 78A 50, 35J05, 35C15, 35P05.

1. Introduction. This work is motivated by optical engineering issues related to fiber optics communication. The problem of wave propagation in a 2-D optical waveguide is considered. The present work is concerned with the development of a framework by which we can understand details of the wave propagation phenomena. Moreover, our work is driven by the need to compute solutions to the wave equation in simulations.

One of the questions we investigate here is the nature of how guided waves are generated from a source in a multimode optical fiber. This is an important issue in design of optical systems. It is also hoped that this work will provide some insight into correct statement of the open waveguide problem.

The main result of this work is a transform theory that allows us to represent solutions to the time harmonic wave equation in a waveguide. In developing the transform technique, we establish a completeness result. As an example of the application of this technique, we will derive an expression for the Green's function. It will be shown that this approach leads to a computationally viable method for accurate simulations. In addition, the expression allows us to separate the energies in the field into guided and non-guided modes. We obtain asymptotic behavior of the non-guided components far away from the source.

We begin with a model for the optical waveguide. We are concerned with timeharmonic wave propagation due to a source. The domain under consideration is all of $\mathbb{R}^{2}$, wherein the field $u(x, z)$ satisfies the Helmholtz equation

$$
\Delta u+k^{2} n(x)^{2} u=f(x, z) \quad(x, z) \in \mathbb{R}^{2} .
$$

The parameter $k$ represents time-frequency of the harmonic behavior, and $n(x)$ is the index of refraction. The source is represented by the function $f(x, z)$. The waveguide lies horizontally as shown in Figure 1.1. The fiber consists of 2 parts, the core $\{|x|<$ $h\}$, and the cladding $\{|x|>h\}$. The index of refraction is $x$-dependent in the core,

${ }^{*}$ The work of R. M. is supported in part by CNR, Italy; the work of F. S. is supported in part by AFOSR under a MURI grant to the University of Delaware, and NSF.

$\dagger$ Dipartimento di Matematica U. Dini, University of Firenze, viale Morgagni 67/A, 50134 Firenze, Italy

$\ddagger$ School of Mathematics, University of Minnesota, Vincent Hall, 206 Church St, Minneapolis, MN 55455 , USA 


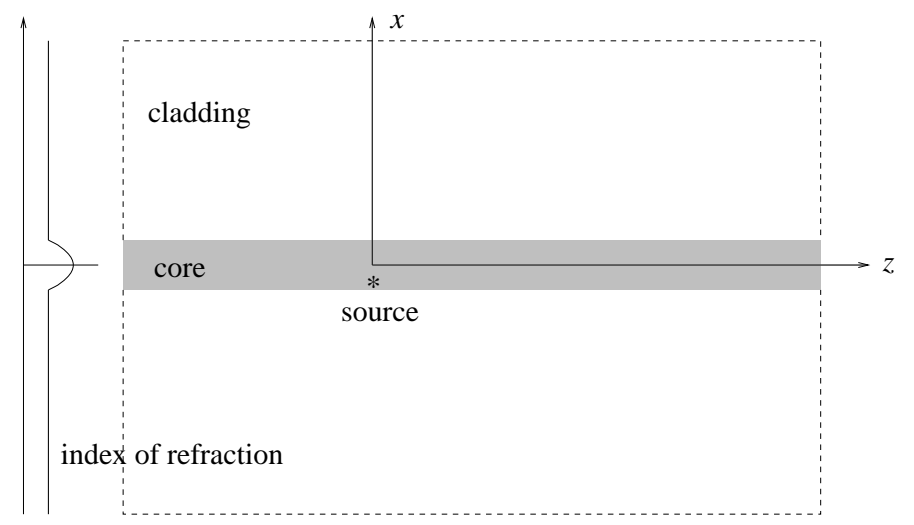

FIG. 1.1. The geometry of the waveguide. The core has an $x$-dependent index of refraction while the cladding has constant properties. The core width is $2 \mathrm{~h}$. For the Green's function, a source is located at $\left(x_{0}, 0\right)$ as indicated in the figure.

and is constant in the cladding. This fact is represented by

$$
n(x)=\left\{\begin{array}{lll}
n_{\mathrm{cl}} & \text { for } & |x|>h \\
n_{\mathrm{co}}(x) & \text { for } & |x|<h
\end{array}\right.
$$

Here, we assume that $n_{\mathrm{co}}(x) \geq n_{\mathrm{cl}}$ and let $n_{0}=n_{\mathrm{co}}(0)$.

In order to complete the description of this problem, we need to prescribe boundary conditions for $x^{2}+z^{2} \rightarrow \infty$. However, it is not clear what the correct boundary conditions are. The work by Nosich and Shestopalov [5] discusses the issue of boundary conditions. The results from the paper indicates that the boundary condition cannot be explicitly prescribed. We defer this issue at this time and instead focus our attention on obtaining an explicit solution that makes physical sense.

The problem under investigation is related to the so-called Pekeris waveguide in ocean acoustics. There are two main differences: (i) the Pekeris waveguide is a halfspace $(x>0)$ with the Dirichlet boundary condition on $x=0$; (ii) the index of refraction is constant in the core region. Wilcox $[8,9]$ investigates the spectrum of the Pekeris operator (using our notation)

$$
A u=-\frac{1}{n(x)^{2}} \Delta u, \text { for } \quad x>0,
$$

with the boundary condition $u(0, z)=0$. We will provide a more detailed relationship between Wilcox's work and our work in the next section.

The result of the present work is closely connected with the work of DeSanto [3]. DeSanto derives an expression for the Green's function of the Pekeris waveguide. The method employed is complex analysis, starting with the assumption of separability of the Green's function in the variables $x$ and $z$, and a representation in terms of a contour integral in the separation parameter. The expressions for the Green's function bear some resemblance to ours. What is different is that our results apply to the more general case where the index of refraction is variable in the core. Moreover, our derivation is based on the transform approach, with rigorous justification, whereas the DeSanto's work is more formal in nature. 
The paper is organized as follows. In the next section, we study the completeness of an associated eigenvalue problem. We attempt to connect our results with those of the classical optical waveguide text such as Snyder and Love [6]. We also discuss our completeness result in the context of Wilcox's work. Section 3 is devoted to the solution of the inhomogeneous problem. We derive an expression for the Green's function. We will also obtain asymptotic results that show how the nonguided part of the field decays along the length of the fiber. Numerical examples are given in Section 4 for the case of the slab waveguide. A discussion section ends the paper.

2. Completeness for an associated eigenvalue problem. The associated eigenvalue problem comes from studying homogeneous solutions of (1.1) of the form

$$
u(x, z)=v(x, \beta) \exp i k \beta z .
$$

We note that $v(x, \beta)$ satisfies

$$
v^{\prime \prime}+k^{2}\left[n(x)^{2}-\beta^{2}\right] v=0 .
$$

We introduce

$$
q(x)=\left\{\begin{array}{lll}
k^{2}\left[n_{0}^{2}-n_{\mathrm{co}}(x)^{2}\right] & \text { for } & |x|<h \\
k^{2}\left(n_{0}^{2}-n_{\mathrm{cl}}^{2}\right) & \text { for } & |x|>h
\end{array} .\right.
$$

Setting $d^{2}=k^{2}\left(n_{0}^{2}-n_{\mathrm{cl}}^{2}\right)$ and

$$
\lambda=k^{2}\left(n_{0}^{2}-\beta^{2}\right),
$$

we arrive at a familiar looking equation for $v$

$$
v^{\prime \prime}+(\lambda-q) v=0 \quad \text { in } \quad \mathbb{R} .
$$

We view $\lambda$ as the 'eigenvalue' parameter.

2.1. Representation of solutions. We consider equation (2.2) under general assumptions on the function $q$ :

(i) $q(-x)=q(x)$ for $x \in \mathbb{R}$ and $q(0)=0$;

(ii) $q$ is non-decreasing on $[0,+\infty)$;

(iii) $q(x)=d^{2}$ for $|x| \geq h$.

Let $Q=\sqrt{\lambda-d^{2}}$; we construct two linearly independent solutions $v_{s}(x, \lambda)$ and $v_{a}(x, \lambda)$ of $(2.2)$ given by

$$
v_{j}(x, \lambda)= \begin{cases}\phi_{j}(h, \lambda) \cos Q(x-h)+\frac{\phi_{j}^{\prime}(h, \lambda)}{Q} \sin Q(x-h), & \text { if } x \geq h \\ \phi_{j}(x, \lambda), & \text { if }|x|<h \\ \phi_{j}(-h, \lambda) \cos Q(x+h)+\frac{\phi_{j}^{\prime}(-h, \lambda)}{Q} \sin Q(x+h), & \text { if } x \leq-h\end{cases}
$$

for $j \in\{s, a\}$. The solutions satisfy the jump conditions

$$
[v( \pm h, \lambda)]=0, \quad\left[v^{\prime}( \pm h, \lambda)\right]=0 .
$$

Here, $\phi_{s}(x, \lambda)$ and $\phi_{a}(x, \lambda)$ are solutions of $(2.2)$ in the interval $(-h, h)$ and satisfy respectively the initial conditions

$$
\begin{aligned}
& \phi_{s}(0, \lambda)=1, \quad \phi_{s}^{\prime}(0, \lambda)=0, \\
& \phi_{a}(0, \lambda)=0, \quad \phi_{a}^{\prime}(0, \lambda)=\sqrt{\lambda} .
\end{aligned}
$$


If $0<\lambda<d^{2}$, in (2.3) we mean $Q=i \sqrt{d^{2}-\lambda}$ and we use the analytic extensions of sine and cosine. Notice that, due to the symmetry of $q, v_{s}(x, \lambda)$ is an even function of $x$, whereas $v_{a}(x, \lambda)$ is odd.

For $\lambda \geq d^{2}, v_{j}(x, \lambda), j \in\{s, a\}$ are bounded. For $\lambda<d^{2}$, we impose

$$
\sqrt{d^{2}-\lambda} \phi_{j}(h, \lambda)+\phi_{j}^{\prime}(h, \lambda)=0, \quad j \in\{s, a\} .
$$

to make the solutions bounded. There will be a finite number of roots $\left\{\lambda_{k}^{j}\right\}_{k=1}^{m_{j}}$, $j \in\{s, a\}$, to (2.6) with associated solutions

$$
v_{j}\left(x, \lambda_{k}^{j}\right), \quad k=1,2, \ldots, m_{j}, \quad j \in\{s, a\} .
$$

These solution decay exponentially for $|x|>h$. For any $\lambda \geq d^{2}$ and $\lambda=\lambda_{k}^{j}$, every bounded solution of $(2.2)$ is spanned by $v_{s}(x, \lambda)$ and $v_{a}(x, \lambda)$.

2.2. Types of solutions. There are three types of solutions of equation (2.2). Each type has a physical interpretation.

Guided modes. For $0<\lambda<d^{2}\left(n_{\mathrm{cl}}<\beta<n_{0}\right)$, (2.2) supports solutions $v_{j}(x, \lambda)$, $j \in\{s, a\}$, that decay exponentially for $|x|>h$ for a finite number of wavenumbers $\beta=\beta_{1}, \beta_{2}, \cdots, \beta_{n_{j}}$. These wavenumbers correspond to eigenvalues $\lambda_{1}, \lambda_{2}, \cdots, \lambda_{n_{j}}$ satisfying the dispersion relation (2.6).

The guided modes, i.e., the solution of the form $u=v(x, \lambda) \exp i k \beta z$ correspond to solutions to Helmholtz equation which are localized near the core, and propagates most of its energy along the core.

Radiation modes. For $d^{2}<\lambda<k^{2} n_{0}^{2}\left(0<\beta<n_{\mathrm{cl}}\right),(2.2)$ supports solutions $v_{j}(x, \lambda)$, $j \in\{s, a\}$, which are bounded and oscillatory. The solutions to Helmholtz equation with these functions correspond to planewaves.

Evanescent modes. For $\lambda>k^{2} n_{0}^{2}$ ( $\beta$ imaginary), (2.2) supports solutions $v_{j}(x, \lambda)$, $j \in\{s, a\}$, which are bounded and oscillatory. The solutions to Helmholtz equation with these functions correspond to waves that decay exponentially in the positive $z$ direction if we take $\beta$ to be positive imaginary. If we take the negative imaginary for $\beta$, the solution decays in the opposite direction.

The literature, see for example [6, 4], suggests that all solutions of Helmholtz equation (1.1) are superpositions of these modes. What is less clear is whether such a representation is complete. Even if it is complete, it is not obvious how one can find the Fourier coefficients in the representation since the solutions do not have finite $L^{2}\left(\mathbb{R}^{2}\right)$ norm. Another potentially troublesome issue is the fact that the evanescent modes blow up either in the positive $z$ or negative $z$ depending on $\beta$.

We resolve the above issues by introducing a transform theory using the solutions $v(x, \lambda)$. This is developed in the next subsections.

2.3. Levitan-Levinson method. In this subsection, we recall the main facts of the Levitan-Levinson transform method as developed in [2], Chapter IX (see also [7], Chapter VI.)

Let $t>h$ and let $\lambda_{n, t}^{j}, n=1,2, \cdots, j \in\{s, a\}$ be the eigenvalues of the SturmLiouville problem for $(2.2)$ in the interval $(0, t)$ with boundary conditions (2.4) for $j=s$ and (2.5) for $j=a$, respectively, and homogeneous Dirichlet boundary condition at $x=t$. From the properties of $v_{j}(x, \lambda), j \in\{s, a\}$, we have that $\lambda_{n, t}^{j}$ are the roots of

$$
v_{j}\left(t, \lambda_{n, t}^{j}\right)=0, \quad j \in\{s, a\} .
$$


We denote by $\psi_{n, t}^{j}(x), n=1,2, \cdots, j \in\{s, a\}$ the corresponding normalized eigenfunctions (normalized on the interval $[-t, t]$ ) and write them as:

$$
\psi_{n, t}^{j}(x)=\sqrt{r_{n, t}^{j}} v_{j}\left(x, \lambda_{n, t}^{j}\right), \quad n=1,2, \cdots, \quad j \in\{s, a\},
$$

where

$$
r_{n, t}^{j}=\left\{\int_{-t}^{t} v_{j}\left(x, \lambda_{n, t}^{j}\right)^{2} d x\right\}^{-1}, \quad n=1,2, \cdots, \quad j \in\{s, a\} .
$$

Let $f \in L^{2}(\mathbb{R})$. Standard Sturm-Liouville theory allows us to write the Fourier expansion for $f$ :

$$
f(x)=\sum_{j \in\{s, a\}} \sum_{n=1}^{+\infty} c_{n, t}^{j} \psi_{n, t}^{j}(x), \quad x \in[-t, t]
$$

where

$$
c_{n, t}^{j}=\int_{-t}^{t} f(x) \psi_{n, t}^{j}(x) d x, \quad j \in\{s, a\} .
$$

If we introduce the transforms

$$
F_{t}^{j}(\lambda)=\int_{-t}^{t} f(x) v_{j}(x, \lambda) d x, \quad j \in\{s, a\}
$$

by $(2.11),(2.9)$ and $(2.12)$, we have

$$
f(x)=\sum_{j \in\{s, a\}} \sum_{n=1}^{+\infty} r_{n, t}^{j} F_{t}^{j}\left(\lambda_{n, t}^{j}\right) v_{j}\left(x, \lambda_{n, t}^{j}\right) .
$$

Let $d \rho_{t}^{j}(\lambda), j \in\{s, a\}$ be the distributions defined by

$$
\left\langle d \rho_{t}^{j}, \eta\right\rangle=\sum_{n=1}^{+\infty} r_{n, t}^{j} \eta\left(\lambda_{n, t}^{j}\right), \quad j \in\{s, a\},
$$

for every $\eta \in C_{0}^{\infty}([0,+\infty))$. Then (2.14) takes the form

$$
f(x)=\sum_{j \in\{s, a\}} \int_{0}^{+\infty} F_{t}^{j}(\lambda) v_{j}(x, \lambda) d \rho_{t}^{j}(\lambda) .
$$

In [2], Chapter IX, it is shown that, as $t \rightarrow+\infty$, then the $F_{t}^{j}$ 's and the $d \rho_{t}^{j}$ 's converge in the proper topologies respectively to the functions $F^{j}, j \in\{s, a\}$, defined by

$$
F^{j}(\lambda)=\int_{-\infty}^{+\infty} f(x) v_{j}(x, \lambda) d x, \quad \lambda \geq 0, \quad j \in\{s, a\},
$$


and to some distributions $d \rho^{j}, j \in\{s, a\}$.

This convergence makes sure that the right-hand side of formula (2.16) is bounded in $L^{2}(\mathbb{R})$ so that we obtain the inverse transform formula

$$
f(x)=\sum_{j \in\{s, a\}} \int_{0}^{+\infty} F^{j}(\lambda) v_{j}(x, \lambda) d \rho^{j}(\lambda) .
$$

Moreover, the Parseval identity

$$
\int_{-\infty}^{+\infty} f(x)^{2} d x=\sum_{j \in\{s, a\}} \int_{0}^{+\infty} F^{j}(\lambda)^{2} d \rho^{j}(\lambda)
$$

holds.

The transform pair (2.17) and (2.18) is the main ingredient needed for analyzing wave propagation problems in 2 -D waveguides. Since $v_{j}(x, \lambda), j \in\{s, a\}$ are well defined in (2.3), what remains is to find the weights $d \rho^{j}(\lambda)$.

2.4. The weight in the transform. We proceed by calculating the distributions $d \rho^{j}, j \in\{s, a\}$.

THEOREM 2.1. Let $f \in L^{2}(\mathbb{R})$ and let $v_{j}$ and $F^{j}, j \in\{s, a\}$ be defined by (2.3) and (2.17) respectively. Let $\left\{\lambda_{k}^{j}\right\}_{k=1}^{m_{j}}, j \in\{s, a\}$ be the roots of (2.6) and let

$$
r_{k}^{j}=\frac{\sqrt{d^{2}-\lambda_{k}^{j}}}{\sqrt{d^{2}-\lambda_{k}^{j}} \int_{-h}^{h} \phi_{j}\left(x, \lambda_{k}^{j}\right)^{2} d x+\phi_{j}\left(h, \lambda_{k}^{j}\right)^{2}}
$$

for $k=1, \ldots, m_{j}, j \in\{s, a\}$.

Then formulae (2.18) and (2.19) hold where $d \rho^{j}, j \in\{s, a\}$, are defined by

$$
\begin{aligned}
\left\langle d \rho^{j}, \eta\right\rangle= & \sum_{k=1}^{m_{j}} r_{k}^{j} \eta\left(\lambda_{k}^{j}\right)+ \\
& \frac{1}{2 \pi} \int_{d^{2}}^{+\infty} \frac{\sqrt{\lambda-d^{2}}}{\left(\lambda-d^{2}\right) \phi_{j}(h, \lambda)^{2}+\phi_{j}^{\prime}(h, \lambda)^{2}} \eta(\lambda) d \lambda, \quad j \in\{s, a\},
\end{aligned}
$$

for every $\eta \in C_{0}^{\infty}([0,+\infty))$.

Proof. We shall give the proof for the case $j=s$. To simplify notations, we will drop the subscript $s$.

By the definition (2.3) of $v(x, \lambda)$ and by $(2.8)$, we have that $\lambda_{n, t}, n=1,2, \cdots$, satisfy the equation

$$
\phi^{\prime}(h, \lambda) \tan \left[(t-h) \sqrt{\lambda-d^{2}}\right]+\phi(h, \lambda) \sqrt{\lambda-d^{2}}=0,
$$

where $\phi(x, \lambda)$ is defined by $(2.2)$ and $(2.4)$.

Isolated eigenvalues. The eigenvalues $\lambda_{k, t}$ which belong to the interval $\left(0, d^{2}\right)$ then satisfy the equation

$$
\phi^{\prime}(h, \lambda) \tanh \left[(t-h) \sqrt{\lambda-d^{2}}\right]+\phi(h, \lambda) \sqrt{d^{2}-\lambda}=0 .
$$


As $t \rightarrow+\infty$, the right-hand side of the latter equation converges uniformly in $\lambda$; hence the $\lambda_{k, t}$ 's smaller than $d^{2}$ converge to the roots $\lambda_{k}, k=1, \ldots, m$ of (2.6). Moreover, by $(2.10)$ we have that

$$
\begin{aligned}
\frac{1}{r_{k, t}}= & \int_{-h}^{h} \phi\left(x, \lambda_{k, t}\right)^{2} d x+ \\
& \frac{1}{\sqrt{d^{2}-\lambda_{k, t}}} \int_{-(t-h) \sqrt{d^{2}-\lambda_{k, t}}}^{(t-h) \sqrt{d^{2}-\lambda_{k, t}}}\left[\phi\left(h, \lambda_{k, t}\right) \cosh s-\frac{\phi^{\prime}\left(h, \lambda_{k, t}\right)}{\sqrt{d^{2}-\lambda_{k, t}}} \sinh s\right]^{2} d s,
\end{aligned}
$$

so that the $r_{k, t}$ 's converge to the values $r_{k}$ 's defined in (2.20).

The continuous spectrum. For fixed $n=1,2, \cdots$, any value $\lambda_{n, t}>d^{2}$ satisfying (2.22) converges to $d^{2}$ as $t \rightarrow+\infty$. Set $\kappa_{n, t}=(t-h) \sqrt{\lambda_{n, t}-d^{2}}$.

If $\phi^{\prime}\left(h, d^{2}\right) \neq 0$, then (2.22) implies that $\kappa_{n, t} \rightarrow n \pi$ and hence $\kappa_{n+1, t}-\kappa_{n, t} \rightarrow \pi$ for $t \rightarrow+\infty$.

By (2.10) and (2.3), we can write

$$
(t-h) r_{n, t}=p_{n, t}\left(\lambda_{n, t}^{j}\right), n=1,2, \cdots,
$$

where

$$
\begin{aligned}
& p_{n, t}(\lambda)= \\
& \frac{\lambda-d^{2}}{t-h} \int_{-h}^{h} \phi(x, \lambda)^{2} d x+\frac{1}{\kappa_{n, t}} \int_{-\kappa_{n, t}}^{\kappa_{n, t}}\left[\sqrt{\lambda-d^{2}} \phi_{j}(h, \lambda) \cos s-\phi^{\prime}(h, \lambda) \sin s\right]^{2} d s
\end{aligned}
$$

for $n=1,2, \cdots$ As $t \rightarrow+\infty, p_{n, t}$ converges uniformly in compact subsets of $[0,+\infty)$ to

$$
p(\lambda)=\frac{\lambda-d^{2}}{\left(\lambda-d^{2}\right) \phi(h, \lambda)^{2}+\phi^{\prime}(h, \lambda)^{2}}, \quad \lambda \in[0,+\infty) .
$$

Now, let $\eta \in C_{0}^{\infty}([0,+\infty))$. If $\eta$ is supported in $\left(0, d^{2}\right)$, by $(2.15)$ we have

$$
\left\langle d \rho_{t}, \eta\right\rangle \rightarrow \sum_{k=1}^{m} r_{k} \eta\left(\lambda_{k}\right)
$$

as $t \rightarrow \infty$.

If the support of $\eta$ is contained in $\left[d^{2},+\infty\right)$, by $(2.15)$ and (2.23), we write

$$
\left\langle d \rho_{t}, \eta\right\rangle=\sum_{\lambda_{n, t} \geq d^{2}} \frac{p_{n, t}\left(\lambda_{n, t}\right) \eta\left(\lambda_{n, t}\right)}{\kappa_{n+1, t}-\kappa_{n, t}}\left(\sqrt{\lambda_{n+1, t}-d^{2}}-\sqrt{\lambda_{n, t}-d^{2}}\right) .
$$

By the uniform convergence of $p_{n, t}$, we have that $d \rho_{t} \rightarrow d \rho$ where

$$
\langle d \rho, \eta\rangle=\frac{1}{\pi} \int_{0}^{+\infty} p\left(d^{2}+\mu^{2}\right) \eta\left(d^{2}+\mu^{2}\right) d \mu .
$$


Finally, by a change of variables, we obtain

$$
\langle d \rho, \eta\rangle=\frac{1}{2 \pi} \int_{d^{2}}^{+\infty} \frac{\sqrt{\lambda-d^{2}}}{\left(\lambda-d^{2}\right) \phi(h, \lambda)^{2}+\phi^{\prime}(h, \lambda)^{2}} \eta(\lambda) d \lambda,
$$

which completes the proof for the case $\phi^{\prime}\left(h, d^{2}\right) \neq 0$.

If $\phi^{\prime}\left(h, d^{2}\right)=0$, since $\phi^{\prime}\left(h, d^{2}\right)$ is an analytic function of $\lambda$, by (2.22) we have that $\kappa_{n, t}$ converges either to $\left(n-\frac{1}{2}\right) \pi$ or $\left(n+\frac{1}{2}\right) \pi$. Then, we repeat the previous argument and we arrive to the same conclusion.

Remark. As we already pointed out in the proof, formula (2.21) does not change in the case where $\phi_{j}\left(h, d^{2}\right)=0$. We notice here that the weight $d \rho^{j}$ becomes singular but still integrable at $\lambda=d^{2}$.

2.5. Relation to Wilcox's work. We can now put the main result in this section in the context of Wilcox's work [8,9]. The work of Wilcox also addresses the question of completeness. However, as we will show, there are some fundamental differences in his results and that of ours. The eigenvalue problem associated with the Pekeris operator is

$$
-\frac{1}{n(x)^{2}} \Delta u=\omega^{2} u, \quad \text { for } \quad x>0
$$

with boundary condition $u(0, z)=0$. The index of refraction here is piecewise constant

$$
n(x)= \begin{cases}n_{0} & \text { for } \quad 0<x<h \\ n_{\mathrm{cl}} & \text { for } \quad x>h\end{cases}
$$

as in a slab waveguide. This problem has a spectrum that can be described as follows. We write $u(x, z)=v(x, \beta) \exp i \beta z$. Then the spectrum consists of (i) $\left\{\omega: \omega>\beta / n_{\mathrm{cl}}\right\}$, and (ii) $\left\{\omega_{m}(\beta), m=1,2, \cdots\right\}$. The latter are dispersion curves satisfying

$$
-\tan \sqrt{\omega^{2} n_{0}^{2}-\beta^{2}} h=\frac{\sqrt{\omega^{2} n_{0}^{2}-\beta^{2}}}{\sqrt{\beta^{2}-\omega^{2} n_{\mathrm{cl}}^{2}}} .
$$

The eigenfunctions for (i) will be denoted by $u(x, z, \beta, \omega)$ while those for (ii) will be denoted by $u_{m}(x, z, \beta):=u\left(x, z, \beta, \omega_{m}(\beta)\right)$. For a fixed $\omega$, the part of the spectrum $0 \leq \beta \leq \omega n_{\mathrm{cl}}$ corresponds the radiation modes, and the point spectrum in $\omega n_{\mathrm{cl}} \leq$ $\beta \leq \omega n_{0}$ corresponds to the guided modes. A sketch of the spectrum is provided in Figure 2.1.

The main results of Wilcox are as follows. Given a function $f(x, z) \in L^{2}\left(\mathbb{R}_{+}^{2}\right)$, it can be expanded as

$$
f(x, z)=\sum_{m=0}^{+\infty} f_{m}(x, z)
$$

where

$$
f_{0}(x, z)=\int_{0}^{+\infty} \int_{\beta / n_{\mathrm{c} 1}}^{+\infty} u(x, z, \beta, \omega) F_{0}(\beta, \omega) d\left(\omega^{2}\right) d \beta
$$




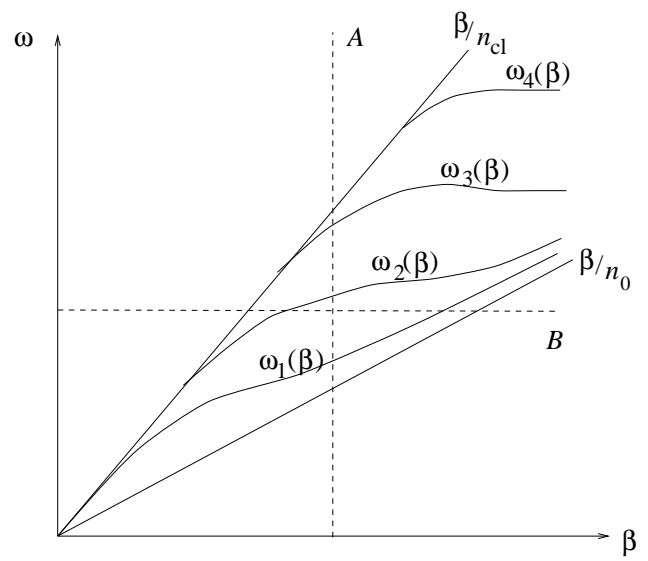

FIG. 2.1. The spectrum of the Pekeris operator in the frequency $(\omega)$ and wavenumber $(\beta)$. The complete spectrum consists of the region $\omega \geq \beta / n_{\mathrm{cl}}$ and the curves $\omega_{m}(\beta)$, which are referred to as dispersion curves.

and

$$
f_{m}(x, z)=\int_{\beta_{m}}^{+\infty} u_{m}(x, z, \beta) F_{k}(\beta) d \beta .
$$

Here, $\beta_{m}$ are the cutoff values, that is, $\omega_{m}(\beta)$ exists for $\beta \geq \beta_{m}$. Simply put, this result states that the eigenfunctions corresponding to the continuous and the 'discrete' spectrum form a complete set in $L^{2}\left(\mathbb{R}_{+}^{2}\right)$. This result is particularly appropriate for analysis of time-dependent propagation as described in [9].

A second result is in a way complementary to our result. We fix $\beta$ and let $v(x, \omega)$ be the solution of

$$
v^{\prime \prime}+\left(\omega^{2} n_{0}^{2}-\beta^{2}\right) v=0 \quad \text { for } \quad x>0,
$$

with $v(0, \omega)=0$. It states that if $f(x) \in L^{2}\left(\mathbb{R}_{+}\right)$, it can be expanded in terms of $v(x, \omega)$ as

$$
f(x)=\sum_{m} F_{m} v\left(x, \omega_{m}(\beta)\right)+\int_{\beta / n_{\mathrm{cl}}}^{+\infty} F(\omega) v(x, \omega) d \sigma(\omega) .
$$

Referring to Figure 2.1, we see that the spectrum in question is the restriction of the Pekeris spectrum along $\beta$ fixed (along line A).

In contrast, our result corresponds to the restriction of the Pekeris spectrum along $\omega$ fixed (line B in Figure 2). It also says that the associated system of eigenfunctions is not complete without the addition of the eigenfunctions corresponding to the evanescent modes.

3. Solution of wave propagation problem. The results of section 2 provides a framework to study the wave propagation problem in a 2-D waveguide. We will derive an expression for the Green's function. The expression allows us to estimate the nonguided components of the solution. We also show how the transform can be used to study a boundary value problem for a half-space waveguide. 
3.1. Green's function of a waveguide. The results of section 2 allow us to construct the Green's function for (1.1) corresponding to the case where no energy is radiated from the farfield $x^{2}+z^{2} \rightarrow \infty$. Here have assumed that the time dependence is $e^{-i k t}$.

Theorem 3.1. Let $f \in C_{0}\left(\mathbb{R}^{2}\right)$ and, for $(x, z),(\xi, \zeta) \in \mathbb{R}^{2}$, let

$$
G(x, z ; \xi, \zeta)=\sum_{j \in\{s, a\}} \int_{0}^{+\infty} \frac{e^{i|z-\zeta| \sqrt{k^{2} n_{0}^{2}-\lambda}}}{2 i \sqrt{k^{2} n_{0}^{2}-\lambda}} v_{j}(x, \lambda) v_{j}(\xi, \lambda) d \rho^{j}(\lambda),
$$

where $v_{j}(x, \lambda)$ and $d \rho^{j}$ are given by (2.3) and (2.21), respectively.

Then the function

$$
u(x, z)=\int_{\boldsymbol{R}^{2}} G(x, z ; \xi, \zeta) f(\xi, \zeta) d \xi d \zeta,(x, z) \in \mathbb{R}^{2},
$$

is of class $C^{1}\left(\mathbb{R}^{2}\right)$ and satisfies (1.1) in the sense of distributions.

Proof. Let

$$
F^{j}(\lambda, z)=\int_{-\infty}^{+\infty} f(\xi, z) v_{j}(\xi, \lambda) d \xi, \quad j \in\{s, a\}
$$

and

$$
U^{j}(\lambda, z)=\int_{-\infty}^{+\infty} u(\xi, z) v_{j}(\xi, \lambda) d \xi, \quad j \in\{s, a\} .
$$

Multiplying (1.1) by $v_{j}(\xi, \lambda)$ and integrating in $\xi$ over $\mathbb{R}$ yields

$$
\begin{aligned}
U_{z z}^{j}(\lambda, z)+ & \int_{-\infty}^{+\infty} u_{\xi \xi}(\xi, z) v_{j}(\xi, \lambda) d \xi+ \\
& k^{2} \int_{-\infty}^{+\infty} n(\xi)^{2} v_{j}(\xi, \lambda) d \xi=F^{j}(\lambda, z), \quad j \in\{s, a\} .
\end{aligned}
$$

Two integrations by parts then give

$$
\begin{gathered}
U_{z z}^{j}(\lambda, z)+\int_{-\infty}^{+\infty}\left[v_{j}^{\prime \prime}(\xi, \lambda)+k^{2} n(\xi)^{2} v_{j}(\xi, \lambda)\right] u(\xi, z) d \xi \\
=F^{j}(\lambda, z), \quad j \in\{s, a\}
\end{gathered}
$$

and hence

$$
U_{z z}^{j}+\left(k^{2} n_{0}^{2}-\lambda\right) U^{j}=F^{j}, \quad j \in\{s, a\},
$$

since $v_{j}(x, \lambda), j \in\{s, a\}$ satisfies (2.2). 
The solution of (3.7), which is outgoing for $0 \leq \lambda<k^{2} n_{0}^{2}$ or decays for $\lambda>k^{2} n_{0}^{2}$ as $|z| \rightarrow+\infty$, is readily found

$$
U^{j}(\lambda, z)=\int_{-\infty}^{+\infty} \frac{e^{i|z-\zeta| \sqrt{k^{2} n_{0}^{2}-\lambda}}}{2 i \sqrt{k^{2} n_{0}^{2}-\lambda}} F^{j}(\lambda, \zeta) d \zeta, \quad j \in\{s, a\},
$$

or, by taking into account (3.3),

$$
U^{j}(\lambda, z)=\int_{-\infty}^{+\infty} \int_{-\infty}^{+\infty} \frac{e^{i|z-\zeta| \sqrt{k^{2} n_{0}^{2}-\lambda}}}{2 i \sqrt{k^{2} n_{0}^{2}-\lambda}} v_{j}(\xi, \lambda) f(\xi, \zeta) d \xi d \zeta, \quad j \in\{s, a\} .
$$

By the inversion formula (2.18), we have

$$
u(x, z)=\sum_{j \in\{s, a\}} \int_{0}^{+\infty} U^{j}(\lambda, z) v_{j}(x, \lambda) d \rho^{j}(\lambda)
$$

hence, by (3.9) and by interchanging the order of integration, we obtain (3.2).

3.2. Decay of nonguided parts. For simplicity, let us consider the Green's function with the source located at $\left(x_{0}, 0\right)$. This corresponds to the point source solution. The expression in (3.1) allows us to separate the the Green's function into 3 components

$$
G(x, z)=G^{g}(x, z)+G^{r}(x, z)+G^{e}(x, z) .
$$

The guided component

$$
G^{g}(x, z)=\sum_{j \in\{s, a\}} \sum_{k=1}^{m_{j}} \frac{e^{i|z| \sqrt{k^{2} n_{0}^{2}-\lambda}}}{2 i \sqrt{k^{2} n_{0}^{2}-\lambda}} v_{j}\left(x, \lambda_{k}^{j}\right) v_{j}\left(x_{0}, \lambda_{k}^{j}\right) r_{k}^{j},
$$

correspond to the solution whose energy is concentrated near the core. The radiated component

$$
G^{r}(x, z)=\sum_{j \in\{s, a\}} \int_{d^{2}}^{k^{2} n_{0}^{2}} \frac{e^{i|z| \sqrt{k^{2} n_{0}^{2}-\lambda}}}{2 i \sqrt{k^{2} n_{0}^{2}-\lambda}} v_{j}(x, \lambda) v_{j}\left(x_{0}, \lambda\right) d \rho^{j}(\lambda),
$$

and the evanescent component

$$
G^{e}(x, z)=\sum_{j \in\{s, a\}} \int_{k^{2} n_{0}^{2}}^{+\infty} \frac{e^{i|z| \sqrt{k^{2} n_{0}^{2}-\lambda}}}{2 i \sqrt{k^{2} n_{0}^{2}-\lambda}} v_{j}(x, \lambda) v_{j}\left(x_{0}, \lambda\right) d \rho^{j}(\lambda) .
$$

are parts of the solution that get radiated away from the source.

In an application, one is interested in how the nonguided part dissipates away from the source and the core, leaving only the guided part in the core. Such knowledge would be relevant for design purposes. We can estimate the $G^{e}$ and $G^{r}$ for a fixed $x$ by studying their integral representations. 


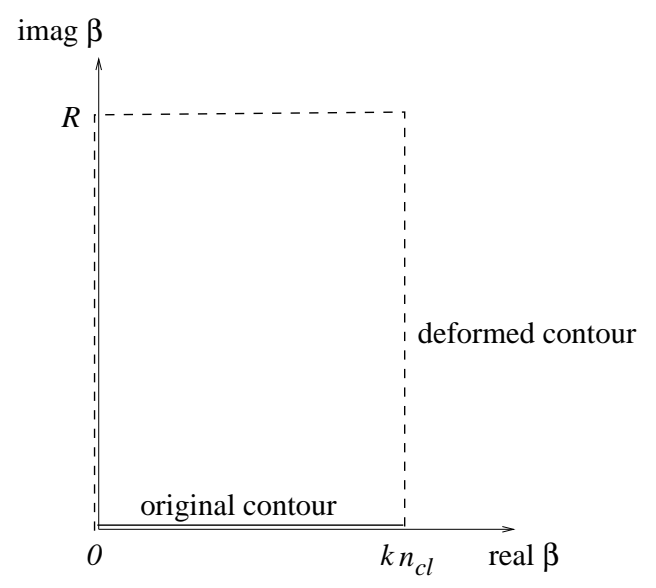

FIG. 3.1. The deformed contour in the complex $\beta$ plane.

We rewrite $G^{e}(x, z)$ by making a change of variable

$$
\beta_{2}=\frac{\sqrt{\lambda-k^{2} n_{0}^{2}}}{k} .
$$

The expression in (3.13) becomes

$$
G^{e}(x, z)=-\left.\sum_{j \in\{s, a\}} \int_{0}^{+\infty} e^{-k \beta_{2}|z|}\left[v_{j}(x, \lambda) v_{j}\left(x_{0}, \lambda\right) \tilde{\rho}^{j}(\lambda)\right]\right|_{\lambda=k^{2}\left(n_{0}^{2}+\beta_{2}^{2}\right)} k d \beta_{2} .
$$

The weight $d \rho^{j}(\lambda)=\tilde{\rho}^{j}(\lambda) d \lambda$ is given in Theorem 2.1. Since the integrand is bounded uniformly in $\beta_{2}$, we can apply Laplace's method [1] and obtain for $|z| \rightarrow \infty$ that

$$
G^{e}(x, z) \approx-\frac{1}{k|z|} v_{j}\left(x, k^{2} n_{0}^{2}\right) v_{j}\left(x_{0}, k^{2} n_{0}^{2}\right) \tilde{\rho}^{j}\left(k^{2} n_{0}^{2}\right) k,
$$

where $v_{j}(x, \lambda)$ have been given in $(2.3)$ and

$$
\tilde{\rho}^{j}\left(k^{2} n_{0}^{2}\right)=\frac{k n_{\mathrm{cl}}}{k^{2} n_{\mathrm{cl}}^{2} \phi_{j}\left(h, k^{2} n_{0}^{2}\right)^{2}+\phi_{j}^{\prime}\left(h, k^{2} n_{0}^{2}\right)^{2}} .
$$

Since $\phi^{\prime}\left(h, k^{2} n_{0}^{2}\right)=O(k)$, and $v\left(x, k^{2} n_{0}^{2}\right)=O(1)$, we see that the factor multiplying $1 / k|z|$ behaves like $O(1)$ uniformly in $k$. Therefore, one can say that for a fixed $x$,

$$
G^{e}(x, z)=O\left(\frac{1}{k|z|}\right) \quad \text { for } \quad z \rightarrow \infty .
$$

We perform a similar analysis for the radiation component. We use the variable $\beta=\sqrt{k^{2} n_{0}^{2}-\lambda} / k$ and rewrite (3.12) as

$$
G^{r}(x, z)=\left.i \sum_{j \in\{s, a\}} \int_{0}^{k n_{\mathrm{cl}}} e^{i k \beta|z|}\left[v_{j}(x, \lambda) v_{j}\left(x_{0}, \lambda\right) \tilde{\rho}^{j}(\lambda)\right]\right|_{\lambda=k^{2}\left(n_{0}^{2}-\beta^{2}\right)} k d \beta
$$


The term multiplying the exponent in the integrand is analytic in $\beta$. We use the Method of Steepest Descent [1]. Deform the contour of integration in the complex $\beta$ plane as shown in Figure 3.1. If we let $R \rightarrow \infty$ in Figure 3.1, the contribution from the vertical parts of the contour are

$$
\begin{aligned}
G^{r}(x, z) & =\left.i \sum_{j \in\{s, a\}} \int_{0}^{\infty} e^{-k \beta_{2}|z|}\left[v_{j}(x, \lambda) v_{j}\left(x_{0}, \lambda\right) \tilde{\rho}^{j}(\lambda)\right]\right|_{\lambda=k^{2} n_{0}^{2}+k^{2} \beta_{2}^{2}} k d \beta_{2} \\
& -\left.i \sum_{j \in\{s, a\}} \int_{0}^{\infty} e^{i k\left(n_{\mathrm{cl}}+i \beta_{2}\right)|z|}\left[v_{j}(x, \lambda) v_{j}\left(x_{0}, \lambda\right) \tilde{\rho}^{j}(\lambda)\right]\right|_{\lambda=k^{2} n_{0}^{2}-k^{2}\left(n_{\mathrm{cl}}+i \beta_{2}\right)^{2}} k d \beta_{2} .
\end{aligned}
$$

We note that as $|z| \rightarrow \infty$, the main contributions in both integrals are near $\beta_{2}=0$. The resulting approximate value of the radiation component is

$$
\begin{aligned}
G^{r}(x, z) & \left.\approx i \sum_{j \in\{s, a\}} \frac{1}{k|z|} k\left[v_{j}(x, \lambda) v_{j}\left(x_{0}, \lambda\right) \tilde{\rho}^{j}(\lambda)\right]\right|_{\lambda=k^{2} n_{0}^{2}} \\
& -\left.i \sum_{j \in\{s, a\}} \frac{1}{k|z|} e^{i k n_{\mathrm{c} 1}|z|} k\left[v_{j}(x, \lambda) v_{j}\left(x_{0}, \lambda\right) \tilde{\rho}^{j}(\lambda)\right]\right|_{\lambda=d^{2}} .
\end{aligned}
$$

By making the same observation as we did in the evanescent case, we can conclude that

$$
G^{r}(x, z)=O\left(\frac{1}{k|z|}\right) .
$$

The upshot of this analysis is that for a fixed $x$, as one looks down the core, the non-guided part of the waves die off like $1 / k|z|$.

3.3. Halfspace problem. The halfspace problem is important in modeling how energy gets launched into a fiber. In this problem, the domain is $z>0$, and we have the boundary condition

$$
u(x, 0)=f(x) .
$$

The boundary condition could represent a light source from a connecting fiber or from a device. We apply the transform method and arrive at the following initial value problem

$$
U_{z z}^{j}+\left(k^{2} n_{0}^{2}-\lambda\right) U^{j}=0, \quad U^{j}(0, \lambda)=F^{j}(\lambda), \quad j \in\{s, a\},
$$

where $F^{j}(\lambda)=\int_{-\infty}^{+\infty} f(x) v^{j}(x, \lambda) d x$. Again, we seek solution that radiates energy towards $z \rightarrow \infty$ and remains bounded. Therefore, the expression for $U^{j}$ is

$$
U^{j}(z, \lambda)=F^{j}(\lambda) e^{i z \sqrt{k^{2} n_{0}^{2}-\lambda}} .
$$

Taking the inverse transform, we get

$$
u(x, z)=\int_{0}^{\infty} F^{j}(\lambda) e^{i z \sqrt{k^{2} n_{0}^{2}-\lambda}} d \rho(\lambda) .
$$

In general, a source $f(x)$ would generate radiation and evanescent modes in addition to the guided modes. The above formula allows one to calculate the field. 
4. Slab waveguide. For a slab waveguide, we have that $n_{\mathrm{co}}(x)=n_{0}>n_{\mathrm{cl}}$. Then, it is straightforward to compute the functions $\phi_{j}(x, \lambda), j \in\{s, a\}$ defined by (2.2) and (2.4) or (2.5).

4.1. Explicit formulae. The expressions for $v_{s}(x, \lambda)$ and $v_{a}(x, \lambda)$ can be drawn from (2.3) where

$$
\begin{array}{ll}
\phi_{s}(x, \lambda)=\cos (x \sqrt{\lambda}), & \phi_{s}^{\prime}(x, \lambda)=-\sqrt{\lambda} \sin (x \sqrt{\lambda}) \\
\phi_{a}(x, \lambda)=\sin (x \sqrt{\lambda}), & \phi_{a}^{\prime}(x, \lambda)=\sqrt{\lambda} \cos (x \sqrt{\lambda}) .
\end{array}
$$

The isolated eigenvalues $\lambda_{k}^{j}, k=1, \ldots, m_{j}, j \in\{s, a\}$ are defined by equations

$$
\sqrt{d^{2}-\lambda_{k}^{s}}-\sqrt{\lambda_{k}^{s}} \tan \left(\sqrt{\lambda_{k}^{s}} h\right)=0, k=1, \ldots, m_{s}
$$

and

$$
\sqrt{d^{2}-\lambda_{k}^{a}} \tan \left(\sqrt{\lambda_{k}^{a}} h\right)+\sqrt{\lambda_{k}^{a}}=0, k=1, \ldots, m_{a}
$$

while

$$
\begin{aligned}
\left\langle d \rho^{s}, \eta\right\rangle= & \sum_{k=1}^{m_{s}} \frac{\sqrt{d^{2}-\lambda_{k}^{s}}}{1+h \sqrt{d^{2}-\lambda_{k}^{s}}} \eta\left(\lambda_{k}^{s}\right)+ \\
& \frac{1}{2 \pi} \int_{d^{2}}^{+\infty} \frac{\sqrt{\lambda-d^{2}}}{\left(\lambda-d^{2}\right) \cos ^{2}(h \sqrt{\lambda})+\lambda \sin ^{2}(h \sqrt{\lambda})} \eta(\lambda) d \lambda, \\
\left\langle d \rho^{a}, \eta\right\rangle= & \sum_{k=1}^{m_{a}} \frac{\sqrt{d^{2}-\lambda_{k}^{a}}}{1+h \sqrt{d^{2}-\lambda_{k}^{a}}} \eta\left(\lambda_{k}^{a}\right)+ \\
& \frac{1}{2 \pi} \int_{d^{2}}^{+\infty} \frac{\sqrt{\lambda-d^{2}}}{\left(\lambda-d^{2}\right) \sin ^{2}(h \sqrt{\lambda})+\lambda \cos ^{2}(h \sqrt{\lambda})} \eta(\lambda) d \lambda,
\end{aligned}
$$

for every $\eta \in C_{0}^{\infty}([0,+\infty))$. These expressions can be used in Theorem 3.1 to obtain an explicit representation of the Green's function for the slab waveguide.

4.2. Numerical examples. The simplicity of the expression for the Green's function in the slab case allows us to numerically evaluate it. We consider a core whose index of refraction is $n_{\text {co }}=2$ with half-width $h=0.2$. The cladding has index of refraction $n_{\mathrm{cl}}=1$. The frequency is taken to be $k=10$.

For the discrete spectrum (guided modes) we solve equations (4.3)-(4.4) for the symmetric and antisymmetric eigenvalues $\lambda_{k}^{s}$ and $\lambda_{k}^{a}$. We employed Newton's method and found 2 symmetric roots $\left\{\lambda_{1}^{s}, \lambda_{2}^{s}\right\}$ and a single antisymmetric root $\left\{\lambda_{1}^{a}\right\}$. We evaluate the weights associated with these eigenvalues using (4.5)-(4.6). Having computed these, we proceed by evaluating the eigenfunctions $v^{s}\left(x, \lambda_{k}^{s}\right), k=1,2$, and $v^{a}\left(x, \lambda_{1}^{a}\right)$. We can calculate the guided part of the Green's function using (3.11).

The continuous spectrum is for $d^{2}<\lambda<\infty$ and the weight associated with this is given in (4.5)-(4.6) for the symmetric and antisymmetric eigenfunctions. In computing the contributions of the radiation and evanescent parts, we use the expressions in 


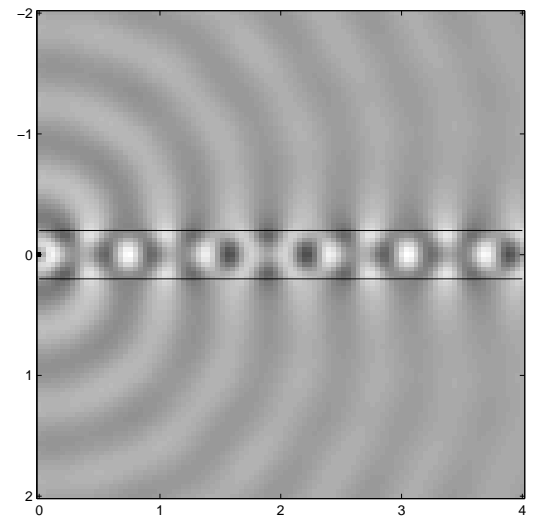

FIG. 4.1. The real part of the Green's function when the source is at $x_{0}=0$. The core/cladding boundaries are indicated by solid lines. Note that most of the energy is confined to the core region.

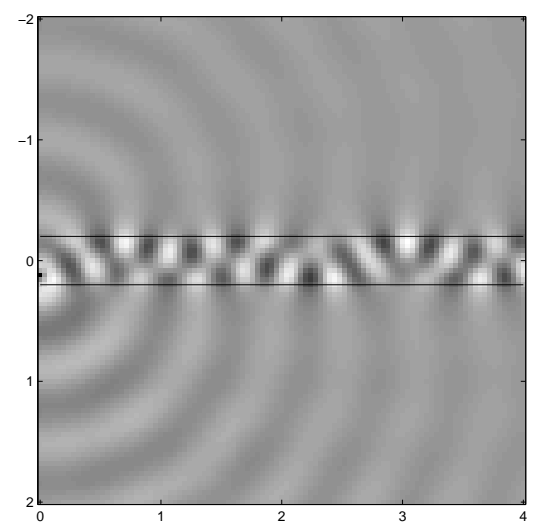

FIG. 4.2. The real part of the Green's function when the source is at $x_{0}=0.1$.

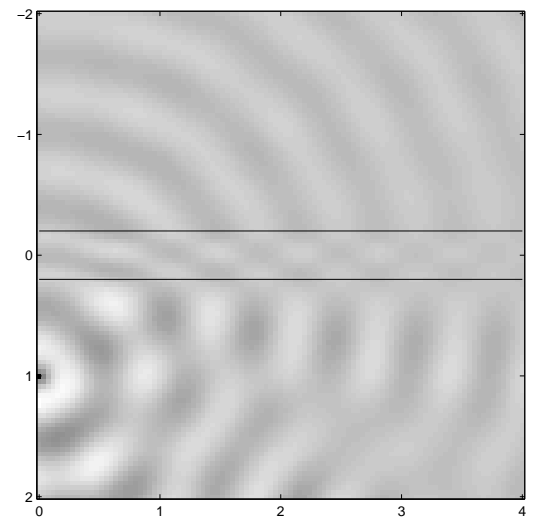

FIG. 4.3. The real part of the Green's function when the source is at $x_{0}=1$. Note that most of the energy is in the nonguided parts. 
(3.15) and (3.14). The trapezoidal rule, with sampling interval $\Delta \beta=0.025$ and $\Delta \beta_{2}=0.1$, is employed. The integral in (3.14) is truncated at $\beta_{2}=12$.

The results of our computations are discussed next. With a source at $x_{0}=0$, we only excite symmetric modes. Figure 4.1 shows the real part of the field $G(x, z)$ for $z \geq 0$. The figure shows that the source generates guided modes, with radiation and evanescent components of the wave decaying away from the source and the core. When the source is at $x_{0}=0.1$, Figure 4.2 , all 3 guided modes are excited. The nonguided components can be seen to decay away from the source and from the core. A final example, Figure 4.3, shows the field when the source is at $x_{0}=1$. Very little guided modes are excited, and we in fact have a scattering situation where most of the waves are either reflected or transmitted across the core.

5. Discussion. In this work, we have constructed a framework for analyzing waveguide problems which is based on a transform theory. The approach allows us to arrive at a representation for the solution of inhomogeneous and half-space problems. The representation is sufficiently simple to allow us to obtain the asymptotic behavior of the nonguided part of the solution. While we applied the method to a 2-D problem, it is possible to apply the approach to 3 -D problems.

For the case of the slab waveguide (piecewise constant indices of refraction), the Green's function is explicit and is very amenable to computation. In the case of general profiles, we believe that there are viable numerical approaches. For example, we can handle the functions $\phi_{j}(x, \lambda)$ in (2.3) numerically using an ODE solver. The discrete spectrum (2.6) can be found numerically using shooting method. Once the discrete spectrum is obtained, we would need to perform a sum over the guided modes, and quadrature over the continuous spectrum.

Acknowledgments. This paper was written while the first author was visiting the School of Mathematics of the University of Minnesota. He would like to thank the School for the kind hospitality. The authors would like to thank also Prof. Fernando Reitich (University of Minnesota) and Allen Cox (Honeywell) for the stimulating discussions on the subject.

\section{REFERENCES}

[1] C. Bender And S. Orszag Advanced Mathematical Methods for Scientists and Engineers McGraw Hill, New York, 1978.

[2] E. A. Coddington And N. Levinson Theory of Ordinary Differential Equations R. E. Krieger Publishing Company, Malabar (Florida) 1984.

[3] J. A. DeSanto, Scalar Wave Theory, Springer-Verlag, Berlin Heidelberg, 1992.

[4] D. Marcuse, Theory of Dielectric Optical Waveguides, Academic Press, New York, 1974.

[5] A. I. Nosich AND V. P. Shestopalov, Radiation conditions and uniqueness theorems for open waveguides, Soviet J. Comm. Tech. Electron., 34 (1989), pp. 107-115.

[6] A. W. Snyder And D. Love, Optical Waveguide Theory, Chapman and Hall, London, 1983.

[7] E. C. Titchmarsh, Eigenfunction Expansions, Second ed., Oxford at the Clarendon Press, Oxford, 1962.

[8] C. Wilcox, Spectral Analysis of the Pekeris operator in the theory of acoustic wave propagation, Archives for Rational Mechanics and Anaysis, 60 (1975), pp. 259-300.

[9] C. Wilcox, Transient electromagnetic wave propagation in a dielectric waveguide, Symposia Mathematica, Vol. XVIII (Convegno sulla Teoria Matematica dell'Elettromagnetismo, INDAM, Rome, 1974), pp. 239-277. Academic Press, London, 1976 